\title{
Response of rats with bilateral superior collicular lesions to change in the ambient visual environment
}

\author{
NIGEL FOREMAN \\ University of Leicester, Leicester, England \\ and \\ CATHERINE THINUS-BLANC \\ CNRS, Marseilles, France
}

\begin{abstract}
Rats with bilateral lesions of the superior colliculus and controls were tested in an apparatus in which the ambient visual environment could be globally modified without changing nonvisual environmental cues. On each of 4 test days, the animals were habituated to either a blank or a patterned visual environment for $5 \mathrm{~min}$ (the habituation phase), removed from the apparatus for $5 \mathrm{~min}$, and then replaced for another $5 \mathrm{~min}$ (the test phase) in either a blank or a patterned environment. When the habituation and test environments were the same (whether patterned or blank), all animals showed a small increase in activity in response to removal and replacement, but this rapidly fell to preremoval levels. When the two environments were visually different, the increase was significantly greater and remained so throughout the test phase, in both the control and the collicular-lesioned groups. In terms of behavioral change, colliculars were therefore as responsive as controls to this form of visual novelty. That the effect was as great in the pattern-to-blank condition as in the blank-to-pattern condition is further evidence that the "missing-stimulus effect" is demonstrable in an open field exploratory paradigm, where it does not depend on the integrity of the superior colliculus.
\end{abstract}

Rats with bilateral collicular lesions are known to be inattentive to a range of environmental stimuli, but especially when unexpected transient events occur in the periphery of the sensory field (Dean \& Redgrave, 1984; Foreman, 1983a; Goodale, Foreman, \& Milner, 1978.) They behave as if unaware of movement and flashing lights, they startle when touched, and they show no evidence of detecting an experimenter's entering the test room (Goodale \& Murison, 1975.) They fail to use distributed spatial cues in navigation tasks (Dean \& Key, 1981; Milner \& Lines, 1983), and they appear not to notice the presence of holes in an apparatus floor, sometimes falling into them (Dean, Pope, Redgrave, \& Donohoe, 1980; Foreman, 1983b.) These effects have been attributed to poor ambient vision (Milner \& Lines, 1983), poor visual scanning and stimulus sampling (see Dean \& Redgrave, 1984, and Milner \& Taylor, 1990), especially of less salient cues (Midgley, Wilkie, \& Tees, 1988), and thus poor acquisition of information from the environment.

The rat superior colliculus receives an extensive input from the retina (Linden \& Perry, 1983), and visual input

\footnotetext{
The authors would like to thank the North Atlantic Treaty Organization for its support of the present work (Grant CRG 890453). We are also grateful to John Ashworth for producing the figures and David Mann for laboratory assistance. Correspondence should be addressed to N. Foreman, Department of Psychology, University of Leicester, University Road, Leicester LE1 7RH, England.
}

to the rat brain must be substantially reduced following collicular ablation, particularly from the visual periphery, a region to which a proportionately large amount of processing is devoted in the rat (see Goodale \& Carey, 1991.) Moreover, since ablation of the colliculus reduces the ability of visual stimulation to arouse the cortex (Dean, Redgrave, \& Molton, 1984), even when the stimulus is in central regions of space (Overton \& Dean, 1988), colliculars might be relatively unaware of novelty occurring in the central visual field. The sparing of central visual functions, including distractibility toward flashing lights, has been reported in collicular rats by Goodale et al. (1978), although their colliculars were less sensitive than controls to central distractors (Dean \& Redgrave, 1984). Indeed, since the animals had been trained to locomote to centrally placed lighted goal doors, they might have been confused, but not genuinely distracted, by the central light flashes.

However, other studies have suggested that colliculars are responsive to unexpected environmental cues, and perhaps as responsive as control animals. They are capable of moving around barriers (Casagrande \& Diamond, 1974), and their behavior takes account of novel objects placed in an open field, since they do not collide with them (Marshall, 1978). They may investigate such objects (Thinus-Blanc, Scardigli, \& Buhot, 1991), although their approach behaviors are erratic and disorganized, and their object contacts are perhaps fortuitous (Foreman, 1980; 
Scardigli, Fabre, \& Thinus-Blanc, 1990). When transported between housing and test rooms (whether or not they remain in their home cages), collicular rats become aroused, as if aware of the change of room (Dean \& Redgrave, 1984; Dean, Pope, \& Redgrave, 1982; Foreman, 1980), and they show nonspecific responses to novel stimuli introduced into runways (Thinus-Blanc \& Lecas, 1985). The elevation of running (hyperactivity) seen in collicular rats in an open-field environment (Foreman, Goodale, \& Milner, 1978; Foreman, 1983b) may also arise from the detection of environmental novelty (Dean et al., 1982).

There is, therefore, evidence for both sensitivity and insensitivity in collicular-lesioned rats to environmental change. However, in many of the test situations that have appeared to yield unambiguous responses to novelty in colliculars, the stimulus changes that were detected involved the introduction of novel multisensory cues. Colliculars may have been responding to nonvisual cuesfor example, the auditory, tactile, or vestibular stimuli produced by cage movement during transportation between rooms. In open-field exploratory tests, they may have responded to olfactory room cues or olfactory and tactile cues from objects placed in an arena. Colliculars fail to reexamine familiar objects appearing in novel spatial locations, but they do respond to new objects in familiar places (Thinus-Blanc et al., 1991), perhaps because they use olfaction rather than vision. Indeed, the relative degradation of visual input following colliculectomy might make animals especially sensitive to stimuli in other modalities. Their hyperactivity on initial exposure to a test arena could arise in part from hyperresponsiveness to handling, for example. It is therefore important to ask whether rats with collicular lesions are as responsive as controls to purely visual change in their environments.

Thinus-Blanc and Foreman (1993) have recently reported a new paradigm that enables the testing of small animals' responses to purely visual novelty, using an apparatus that is constructed so that the visual environment can be altered, leaving all other aspects of the test situation unchanged. Animals are habituated to a first environment, removed from the apparatus, and then replaced in an environment that may be either the same as the first or visually different. Control animals' activity increases after replacement, in all conditions, but the increase is larger and more persistent when the first and second environments are visually different. In the present study, we investigated this phenomenon in collicular-lesioned rats.

Another issue addressed in this study was whether animals with collicular lesions would display the "missingstimulus effect" - that is, whether, as in the controls, their response to the absence of an expected salient stimulus (patterning in the environment) would be as great as would their response to the unexpected appearance of a novel salient stimulus. Responses to absent expected stimuli are extremely weak in control animals tested in runways
(Foreman \& Thinus-Blanc, 1987), but we have found that in open-field environments, they show as great an increase in activity when switched from a patterned to a blank environment as when switched from blank to pattern (Thinus-Blanc \& Foreman, 1993, Experiment 2). Since this requires novelty detection, it was of interest to know whether the phenomenon would occur in animals with collicular lesions.

\section{METHOD}

\section{Subjects}

The subjects were 11 male hooded rats of the Sprague-Dawley strain, weighing $250 \mathrm{~g}$, housed in standard laboratory cages in a colony room (lights on 7:00 a.m. to 7:00 p.m.). They had ad-lib access to food (laboratory chow) and water.

\section{Apparatus}

The apparatus is described in another publication (Thinus-Blanc \& Foreman, 1993, Experiment 2). In brief, it consisted of a square, hardboard arena measuring $85 \times 85 \mathrm{~cm}$ with walls $49 \mathrm{~cm}$ high, the inner facing surfaces all painted a matt gray. Within the outer walls was a clear Perspex inner arena, $84 \times 84 \mathrm{~cm}$ and $48.5 \mathrm{~cm}$ high, that left a $0.5-\mathrm{cm}$ space between the inner and outer surfaces. Into this space (in the sides and base) could be inserted stimulus cards. The cards had a checkered pattern, with $7 \times 7 \mathrm{~cm}$ black and white squares alternating across the entire surface. By inserting these checkered cards behind the four sides and the base of the apparatus, one could alter the entire visual field; however, to the rat (which always explored the Perspex surface) all auditory, tactile, and olfactory cues in the environment remained constant. An overhead video camera was located above the arena, leading to a video recorder and monitor in a corner of the room (out of sight of the animal). The apparatus was placed at floor level in a 5-msquare room and was illuminated by a $150-\mathrm{W}$ photographic flood lamp directed at the white ceiling, giving a uniform illumination of $40 \mathrm{~lx}$ across the apparatus surface. A ventilator fan masked extraneous noises. The humidity in the test room was $60 \%$.

\section{Procedure}

Three weeks prior to testing, 3 rats were given bilateral superior collicular lesions under deep barbiturate anesthesia. They were placed in a stereotaxic frame, with the upper incisor bar and the interaural line in the same horizontal plane. A Teflon-insulated radiofrequency electrode with a 1-mm tip exposure, connected to a Radionics lesion maker, was lowered into each colliculus in turn at a position $6.2 \mathrm{~mm}$ posterior to the bregmoidal suture, $1.3 \mathrm{~mm}$ lateral to the midline, and $3.2 \mathrm{~mm}$ below the cortical surface, the tissue being heated to $70^{\circ} \mathrm{C}$ for $90 \mathrm{sec}$. Surgical details have been given in earlier publications (e.g., Foreman, 1983a, 1983b). Four control rats were given small cortical control lesions of tissue overlying the superior colliculus, but since these animals behaved as the other 4 controls, which were not operated on, the 8 control rats will be considered as a single group for the purpose of data analysis. Note that in view of the robust nature of the hyperactivity effect in rats given deep bilateral collicular lesions (Foreman et al., 1978), a larger collicular group could not be justified.

The rats were tested individually in each of the four test conditions, spaced $24 \mathrm{~h}$ apart. The order in which the animals received conditions was decided by the toss of a coin. Every test condition consisted of (1) a first 5-min observation period (habituation phase), (2) removal of the animal from the apparatus for $5 \mathrm{~min}$, and then (3) replacement of the animal in the arena for another $5 \mathrm{~min}$ (test phase). In the habituation and test phases, the animal was observed 
by an experimenter seated at the video monitor, and the animal's activity was recorded on video tape. Lines drawn on the screen of the video monitor divided it into 25 squares, each representing 16.8 $\times 16.8 \mathrm{~cm}$ of apparatus floor. These were used to record the animal's activity, one line crossing being scored each time the animal's front paws crossed a line on the video monitor screen.

The four experimental conditions were characterized according to the stimuli present in the habituation and test phases. In the first, the environment was a homogeneous gray in both conditions (blankto-blank). In the second, check-patterned stimulus cards were inserted behind the Perspex in both the habituation and the test phases (check-to-check). These two conditions will be referred to collectively as no-change conditions. In the two change conditions, the apparatus was either blank in the habituation phase and checkered in the test phase (blank-to-check) or checkered in the habituation phase and blank in the test phase following removal of the checkered cards from the apparatus (check-to-blank condition).

On completion of the experiment, the animals were used in subsequent studies but were eventually sacrificed with a lethal dose of barbiturate anesthetic and transcardially perfused with a $10 \%$ formal saline solution; the brains were removed and sectioned on a freezing microtome. Sections were taken at $30_{-\mu}$ intervals throughout the lesions, and every 10th section was stained with cresyl fast violet. The sections were magnified and projected with a Kodak enlarger, and the extent of each lesion was determined with reference to a standard stereotaxic atlas (König \& Klippel, 1963.) The sections were also examined microscopically for gliosis and cell loss in the dorsal midbrain.

\section{RESULTS}

\section{Histology}

A typical collicular lesion (Animal A2) is shown in Figure 1 , which illustrates the extensive damage that was caused to both superficial and deep laminae of the superior colliculus in each case. There was minor tissue sparing in posteromedial colliculus (representing the upper visual periphery), although since the lesions interrupted the brachium of the superior colliculus bilaterally and destroyed intertectal pathways, any remaining tissue (in which there was dense gliosis) is unlikely to have remained functional. Minor damage occurred to the pretectum and posterior thalamic nuclei, although the only structure extensively damaged in each case was the superior colliculus.

\section{Behaviors}

Activity scores in the habituation phase (Figure 2 ) were subjected to a 2 (groups) $\times 2$ (pattern/no pattern) $\times 5$ (minutes) analysis of variance (ANOVA), which revealed that the collicular group was, as expected, more active than controls $[F(1,9)=9.42, p<.02]$. This was so across all 5 minutes in both pattern and blank conditions, since no interaction term approached statistical significance. Activity decreased significantly across successive minutes $[F(4,36)=11.06, p<.001]$. For the principle analyses of responses to change, each animal's linecrossing score in each minute (6-10) of the test session was expressed as a multiple of that animal's average score in Minutes 1-5 of the habituation phase. This was done in order to adjust postreplacement scores according to the animals' individual differences in baseline activity. This was particularly important in view of the colliculars'
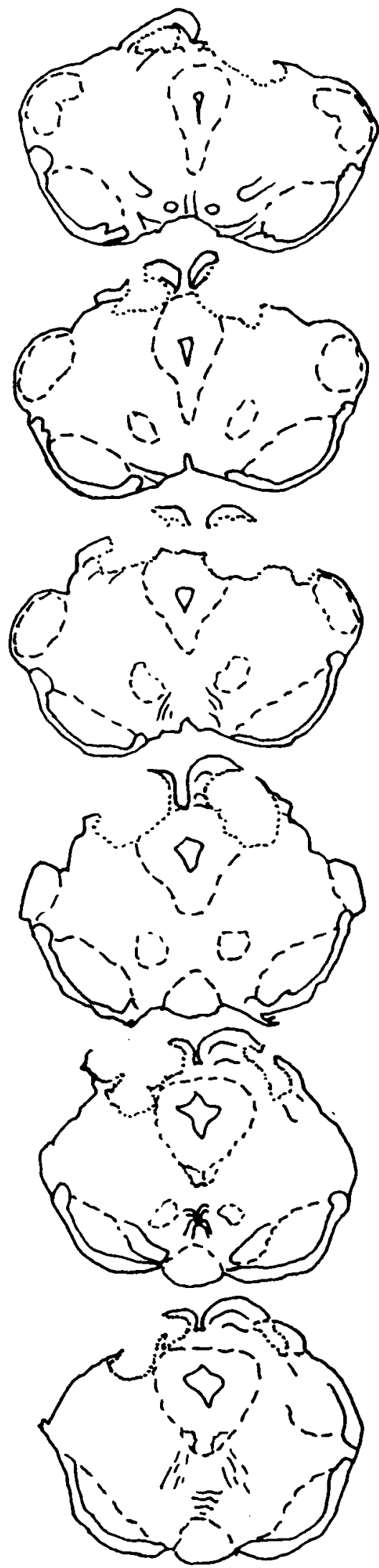

Figure 1. A typical lesion of the superior colliculus, shown in coronal section. 


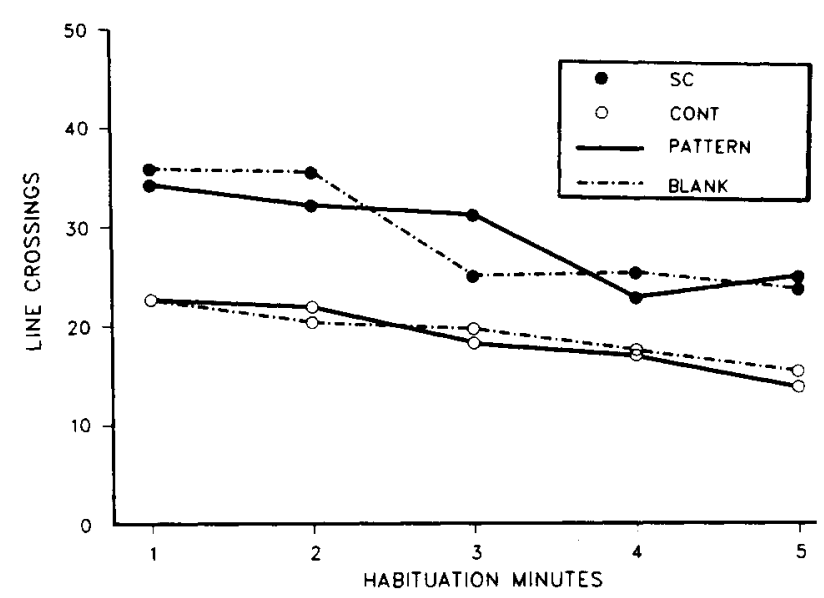

Figure 2. Mean activity level (screen lines crossed) in the habituation phase (Test Minutes 1-5) in collicular and control rats, in patterned and blank environments.

higher baseline activity level. The resulting values for Test Minutes 6-10 were averaged for the control and collicular groups in each of the four test conditions and plotted in Figure 3. The data were entered into a 2 (groups) $\times$ 2 (change/no change) $\times 2$ (pattern $/$ no pattern) $\times 5$ (test minutes) ANOVA, and significant effects were further examined in orthogonal comparisons. The pattern variable in the analysis refers to whether or not the animal was habituated in a blank or a checkered environment.

The analysis revealed that in both no-change conditions, simple removal and replacement produced a temporary revival of locomotor activity, above the mean activity level during the habituation phase (which is represented by 1.0 on the vertical scale in Figure 3) for just the first postreplacement minute. This quickly fell to below the habituation phase average; the factor of test minutes was highly significant $[F(4,36)=40.9, p<.001]$. However, in both groups in the change conditions, the revival of activity was stronger, the change factor being the only other significant variable in the analysis $[F(1,9)=13.95, p<$ $.01]$, with activity in the change conditions exceeding that in the no-change conditions across the whole test phase. Neither the pattern factor $[F(1,9)=.06, p>.05]$ nor the pattern $\times$ change interaction $[F(1,9)=.10, p>.05]$ approached significance.

Since the revival of activity in Postreplacement Minute 1 was expressed as a multiple of the mean activity in the 5 minutes of the habituation phase, the equivalent proportional increase in both groups reflects a greater absolute increase in line crossings in the collicular group. To examine this further, we compared the absolute increase in the number of lines crossed in the change and no-change conditions in the two groups (Figure 4). When replaced in the same environment as that in which they were habituated (no change), both groups increased their activity to the same degree (Mann-Whitney $U$ test: $N 1=3, N 2=8 ; U=7, p>.05$ ), but when replaced in a different environment (change), the colliculars' ac- tivity increased more than the controls' $(U=2, p<$ .05 ). Indeed, the latter result would have been clearer but for 1 unusually active control animal; when data were analyzed with this subject excluded, the difference between colliculars and controls was highly significant $(N 1=3$, $N 2=7 ; U=0, p<.01$ ).

\section{DISCUSSION}

It is clear from the results that the behavior of rats with superior collicular ablations changes in response to environmental change, and that it does so to a greater degree than controls'. This is surprising, in view of the reduction in overall visual input to the brain that must result from interruption of the retinotectal pathway, the usual characterization of colliculars as "inattentive," and the reduction in visual sensitivity and use of visuospatial cues that has been demonstrated in colliculars in a number of paradigms (Dean \& Redgrave, 1984; Milner \& Lines, 1983). Clearly, in the present test we used a behavioral measure in a freely moving animal, involving change within both the central and the peripheral visual fields, and therefore we cannot identify the regions of visual space within which novel environmental cues were detected by these colliculectomized subjects.

The initial hyperactivity of this group of collicular rats is consistent with many earlier studies (Dean et al., 1982; Foreman et al., 1978; Midgley, Prkachin, \& Tees, 1981; see also Foreman \& Stevens, 1987). Furthermore, although both groups increased their activity in the postreplacement period in the change conditions to approximately 1.5 times the average level seen in the habituation period, the absolute increase in line crossings was substantially greater in colliculars than in controls (see Results and Figure 3 ). Since visual environmental change can revive collicular hyperactivity, our data strongly suggest that colliculars' elevated activity when they are ini-

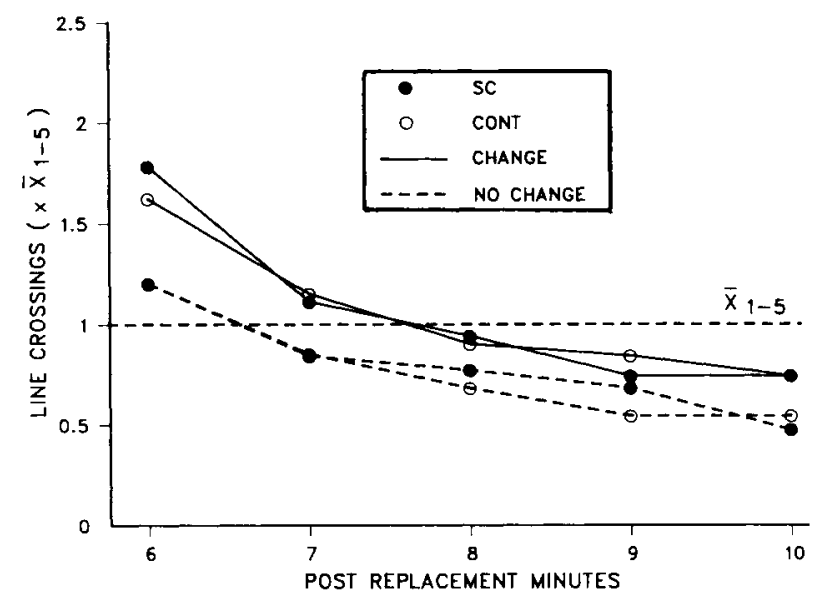

Figure 3. Mean activity level in collicular and control rats in Minutes 6-10 (postreplacement, test phase), expressed for each minute as a multiple of mean activity in Minutes 1-5 (habituation phase). 


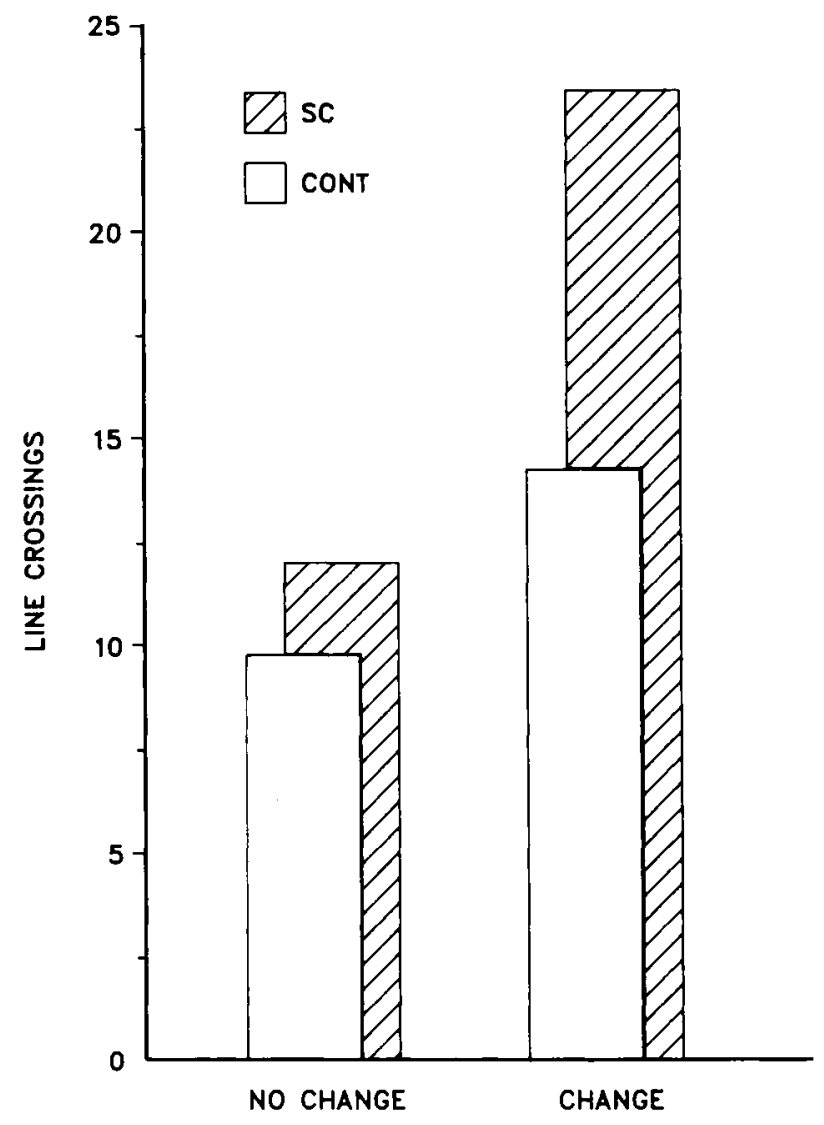

Figure 4. Absolute increase in activity level in Minute 6 (immediately postreplacement), compared with Minute 5 (the final minute of the habituation phase) in collicular and control subjects in change and no-change conditions.

tially tested in novel open-field arenas arises, at least in part, from the animals' detection of visual novelty. This has not been shown previously, since no test situation has been available that allows the selective manipulation of visual cues. Kallman and Isaac (1980) reported that collicular hyperactivity was greater in the light than in the darkness, and though Murison (1977) reported collicular hyperactivity in darkness, it is possible that colliculars in that study were responding to missing visual stimuli; sudden placement in darkness might give rise to increased activity in the same way that replacement in an unexpectedly blank environment did in the present subjects. Although our present results do not allow us to compare among sensory modalities, it is likely that the hyperactivity that is aroused in colliculars when they are moved from room to room or from their home cages to a test arena results from the detection of changes in the visual environment and is unlikely to be due to nonvisual influences alone (cf. Dean et al., 1982). We cannot be as certain about colliculars' use of vision in other exploratory paradigms, such as the exploration of objects introduced into open-field arenas, although they appear to possess a degree of sensitivity to novel visual events that makes the use of vision likely in such circumstances. The present paradigm (Thinus-Blanc \& Foreman, 1993) might be adapted to investigate the use of vision in object investigation by having transparent, airtight containers that remain in fixed places in an arena, into which visually interesting objects could be introduced.

The present data are consistent with earlier models put forward to explain why collicular rats are hyperactive (cf. Foreman et al., 1978). It is proposed that novelty in the environment (including visual novelty) creates arousal and exploration by increasing uncertainty within the animal's environment (Berlyne, 1960), and that a function of exploration is to resolve uncertainty. The specific exploratory acts in which the normal animal engages during open-field exploration (such as rearing vertically, sniffing the floor, or scrutinizing particular cues) are incompatible with continuing locomotion, and thus the nonspecific activity that is provoked by environmental novelty becomes restricted. Such specific exploratory acts (whether under the animal's volition, or externally produced by punctate transient stimulation such as light flashes) may exert an inhibitory influence on the motor system, perhaps via descending fibers from the superior colliculus to the brain stem (Foreman et al., 1978). Colliculars, which notably fail to engage in such specific exploratory behaviors (Dean et al., 1980; Foreman et al., 1978; Thinus-Blanc \& Lecas, 1985), do not produce such inhibitory signals, and their activity is consequently unrestrained and excessive. Note, however, that within this model, the initial detection of gross environmental novelty (and consequent arousal and locomotion) is assumed to result from processing in structures other than the superior colliculus, a supposition that also finds support in the present data. It is also worth noting that collicular rats in this study were tested after a short postoperative interval; even greater sensitivity to environmental changes might be evident after a longer interval, when nonspecific aspects of orienting show a degree of recovery (see Milner \& Taylor, 1990).

Regarding the missing-stimulus effect, the present results provide further evidence that it occurs in an openfield arena-that is, that animals are as affected by a change from a patterned to a blank environment as they are by a change from a blank to a patterned environment (Thinus-Blanc \& Foreman, 1993, Experiment 2). In runways, conversely, the effect is extremely difficult to demonstrate, and rats rarely give orienting responses to nonappearing expected stimuli, despite giving reliable distraction responses to appearing, unexpected stimuli (Foreman \& Thinus-Blanc, 1987). It seems clear that exploratory behaviors in an open-field arena (under the animal's voluntary control) differ in important respects from the reflexive orientations toward sudden punctate stimuli seen in runway distraction studies. Missing stimuli are detected in runways, since stimulus omission disinhibits responses to subsequent distractor appearances (Foreman, Save, Thinus-Blanc, \& Buhot, 1992). Whether the phenome- 
non is colliculus dependent in runways is unknown, although it is clear from the present data that in an openfield environment, responding to the unexpected absence of an environmental pattern does not depend on the integrity of the superior colliculus.

\section{REFERENCES}

Berlyne, D. E. (1960). Conflict, arousal and curiosity. New York: McGraw-Hill.

Casagrande, V. A., \& Diamond, I. T. (1974). Ablation study of the superior colliculus in the tree shrew (Tupaia glis). Journal of Comparative Neurology, 156, 207-238.

DEAN, P., \& KEY, D. (1981). Spatial deficits on radial maze after large tectal lesions in rats: Possible role of impaired scanning. Behavioral \& Neural Biology, 32, 170-190.

Dean, P., Pope, S., \& Redgrave, P. (1982). Influence of novelty on locomotor hyperactivity after lesions of superior colliculus in rats. Behavioural Brain Research, 5, 213-218.

Dean, P., Pope, S., Redgrave, P., \& Donohoe, T. (1980). Superior colliculus lesions in rat abolish exploratory head-dipping in hole-board test. Brain Research, 197, 571-576.

Dean, P., Redgrave, P. (1984). The superior colliculus and visual neglect in rat and hamster: 1. Behavioural evidence. Brain Research Reviews, 8, 129-141.

Dean, P., Redgrave, P., \& Molton, L. (1984). Visual desynchronization of cortical EEG impaired by lesions of superior colliculus in rats. Journal of Neurophysiology, 52, 625-637.

FOREMAN, N. (1980). Spatial and attentional functions of the midbrain visual system. Unpublished doctoral dissertation, University of Nottingham.

ForemAN, N. (1983a). Distractibility following simultaneous bilateral lesions of the superior colliculus or medial frontal cortex in the rat. Behavioural Brain Research, 8, 177-194.

ForemAN, N. (1983b). Head-dipping in rats with superior collicular, medial frontal cortical and hippocampal lesions. Physiology \& Behavior, 30, 711-717.

Foreman, N., Goodale, M. A., \& Milner, A. D. (1978). Nature of postoperative "hyperactivity" following lesions of the superior colliculus in the rat. Physiology \& Behavior, 21, 157-160.

Foreman, N., Save, E., Thinus-Blanc, C., \& Buhot, M.-C. (1992). Visually guided locomotion, distractibility, and the missing-stimulus effect in hooded rats with unilateral or bilateral lesions of parietal cortex. Behavioral Neuroscience, 106, 529-538.

Foreman, N., \& STEvens, R. (1987). Relationships between the superior colliculus and hippocampus: Behavioral and neural considerations. Behavioral \& Brain Sciences, 10, 101-151.

Foreman, N., \& Thinus-Blanc, C. (1987). Weakness of the "missingstimulus effect" in hooded rats: Gross asymmetry in the Sokolovian orienting response. Psychobiology, 15, 265-271.

Goodale, M. A., \& CareY, D. P. (1991). The role of the cerebral cor- tex in visuomotor control. In B. E. Kolb \& R. C. Tees (Eds.), The cerebral conex of the rat (pp. 309-340). Cambridge, MA: MIT Press.

Goodale, M. A., Foreman, N. P., Milner, A. D. (1978). Visual orientation in the rat: A dissociation of deficits following cortical and collicular lesions. Experimental Brain Research, 31, 445-457.

Goodale, M. A., Murison, R. C. C. (1975). The effects of lesions of the superior colliculus on locomotor orientation and the orienting reflex in the rat. Brain Research, 88, 243-261.

Kallman, M. D., IsaAC, W. (1980). Distuption of illumination dependent activity by superior colliculus destruction. Physiology \& Behavior, 25, 45-47.

König, J. F. R., KLIPPEL, R. A. (1963). The rat brain: A stereotaxic atlas of the forebrain and lower parts of the brain stem. Baltimore, MD: Williams \& Wilkins.

Linden, R., \& Perry, V. H. (1983). Massive retinotectal projections in rats. Brain Research, 272, 145-149.

Marshale, J. F. (1978). Comparison of the sensorimotor dysfunctions produced by damage to the lateral hypothalamus or superior colliculus in the rat. Experimental Neurology, 58, 203-217.

Midgley, G. C., Prkachin, K. M., \& Tees, R. C. (1981). Explor atory behavior of rats following visual cortical and subcortical lesions. Physiological Psychology, 9, 152-156.

Midgley, G. C., Wilkie, D. M., \& Tees, R. C. (1988). Effects of superior colliculus lesions on rats' orienting and detection of neglected visual cues. Behavioral Neuroscience, 102, 93-100.

Milner, A. D., Lines, C. R. (1983). Stimulus sampling and the use of distal visual cues in rats with lesions of the superior colliculus. Behavioural Brain Research, 8, 387-401.

Milner, A. D., TAYLOR, M. J. (1990). Auditory orienting and detection in rats following lesions of the superior colliculus. Behavioural Brain Research, 37, 293-296.

Murison, R. C. C. (1977). Superior colliculus lesions in rats. Unpublished doctoral dissertation, University of Leicester.

Overton, P., \& DeAN, P. (1988). Detection of visual stimuli after lesions of the superior colliculus in the rat; deficit not confined to the far periphery. Behavioural Brain Research, 31, 1-15.

Scardigli, P., Fabre, J. C., \& Thinus-Blanc, C. (1990). The effects of superior colliculus lesions on reactions to novelty in the hamster. Behavioural Brain Research, 36, 27-32.

Thinus-Blanc, C., Foreman, N. (1993). A rapid test of rodents' vision using a modified open field apparatus. Physiology \& Behavior, 53, $1003-1006$.

Thinus-Blanc, C., Lecas, J. C. (1985). Effects of collicular lesions in the hamster during visual discrimination: An analysis from computer-video actograms. Quarterly Journal of Experimental Psychology, 37B, 213-233.

Thinus-Blanc, C., Scardigli, P., \& Buhot, M.-C. (1991). The effects of superior colliculus lesions in hamsters: Feature detection versus spatial localization. Physiology \& Behavior, 49, 1-6.

(Manuscript received November 16, 1992; revision accepted for publication March $15,1993$. 